\title{
Gradient nonlinearity correction in liver DWI using motion-compensated diffusion encoding waveforms
}

\author{
Sean McTavish ${ }^{1}$ (D) Anh T. Van ${ }^{1} \cdot$ Johannes M. Peeters ${ }^{2} \cdot$ Kilian Weiss $^{3} \cdot$ Marcus R. Makowski $^{1} \cdot$ Rickmer F. Braren $^{1}$. \\ Dimitrios C. Karampinos ${ }^{1}$
}

Received: 9 June 2021 / Revised: 25 November 2021 / Accepted: 26 November 2021 / Published online: 11 December 2021

(c) The Author(s) 2021

\begin{abstract}
Objective To experimentally characterize the effectiveness of a gradient nonlinearity correction method in removing ADC bias for different motion-compensated diffusion encoding waveforms.

Methods The diffusion encoding waveforms used were the standard monopolar Stejskal-Tanner pulsed gradient spin echo (pgse) waveform, the symmetric bipolar velocity-compensated waveform (sym-vc), the asymmetric bipolar velocity-compensated waveform (asym-vc) and the asymmetric bipolar partial velocity-compensated waveform (asym-pvc). The effectiveness of the gradient nonlinearity correction method using the spherical harmonic expansion of the gradient coil field was tested with the aforementioned waveforms in a phantom and in four healthy subjects.

Results The gradient nonlinearity correction method reduced the ADC bias in the phantom experiments for all used waveforms. The range of the ADC values over a distance of $\pm 67.2 \mathrm{~mm}$ from isocenter reduced from $1.29 \times 10^{-4}$ to $0.32 \times 10^{-4}$ $\mathrm{mm}^{2} / \mathrm{s}$ for pgse, $1.04 \times 10^{-4}$ to $0.22 \times 10^{-4} \mathrm{~mm}^{2} / \mathrm{s}$ for sym-vc, $1.22 \times 10^{-4}$ to $0.24 \times 10^{-4} \mathrm{~mm}^{2} / \mathrm{s}$ for asym-vc and $1.07 \times 10^{-4}$ to $0.11 \times 10^{-4} \mathrm{~mm}^{2} / \mathrm{s}$ for asym-pvc. The in vivo results showed that ADC overestimation due to motion or bright vessels can be increased even further by the gradient nonlinearity correction.

Conclusion The investigated gradient nonlinearity correction method can be used effectively with various motion-compensated diffusion encoding waveforms. In coronal liver DWI, ADC errors caused by motion and residual vessel signal can be increased even further by the gradient nonlinearity correction.
\end{abstract}

Keywords Diffusion-weighted imaging $\cdot$ Gradient nonlinearity $\cdot$ Motion compensation $\cdot$ ADC mapping $\cdot$ Liver imaging

$\begin{array}{ll}\text { Abbreviations } \\ \text { DWI } & \text { Diffusion-weighted imaging } \\ \text { ADC } & \text { Apparent diffusion coefficient } \\ \text { gnl } & \text { Gradient nonlinearity } \\ \text { EPI } & \begin{array}{l}\text { Echo planar imaging } \\ \text { ODGD }\end{array} \\ & \begin{array}{l}\text { Optimized diffusion-weighting gradient wave- } \\ \text { form design }\end{array} \\ \text { pgse } & \begin{array}{l}\text { Pulsed gradient spin echo } \\ \text { sym-vc }\end{array} \\ \text { Symmetric velocity compensated }\end{array}$

Sean McTavish

sean.mctavish@tum.de

1 Department of Diagnostic and Interventional Radiology, School of Medicine, Klinikum rechts der Isar, Technical University of Munich, Munich, Germany

2 Philips Healthcare, Best, The Netherlands

3 Philips Healthcare, Hamburg, Germany asym-vc Asymmetric velocity compensated

asym-pvc Asymmetric partial velocity compensated

\section{Introduction}

Diffusion-weighted imaging (DWI) remains a valuable tool in liver lesion detection and there is an ongoing interest in ADC mapping for tumor staging and therapy monitoring [1, 2]. However, cardiac and respiratory motion from the subject during the standard monopolar Stejskal-Tanner-pulsed gradient spin echo (pgse) diffusion encoding gradients can cause intravoxel dephasing and therefore signal loss [3-8]. Since the apparent diffusion coefficient (ADC) is proportional to the diffusion-induced signal decay when diffusion encoding is applied, any undesired motion-induced signal loss will, therefore, cause an overestimation of the ADC. The close proximity of the left liver lobe to the heart makes 
the left liver lobe especially susceptible to cardiac motioninduced signal loss [7].

Respiratory-triggered scans are most frequently used to overcome issues caused by the breathing motion of the subject in liver DWI [3, 4, 9-12]. Cardiac-triggered scans have also been proposed to overcome the issues caused by cardiac motion. However, cardiac-triggered DWI scans are associated with low acquisition efficiency and require the optimisation of the trigger delay at each individual subject [13]. In addition, it has been recently suggested that a single trigger delay might not be adequate for removing artifacts induced by cardiac motion and vessel pulsation throughout the whole liver $[14,15]$. Therefore, motion-compensated diffusion gradient encoding waveforms have recently been proposed as alternatives for motion-robust liver DWI [15-24].

The simplest motion-compensated diffusion encoding waveform that has been proposed is a symmetric bipolar waveform (sym-vc). An example of the sym-vc waveform design is given in Fig. 1b. In this waveform, the gradient first moment $\left(m_{1}=\gamma \int t G(t) d t\right)$ is zero, leading to no phase accumulation and hence no signal loss when spins move with constant velocities during diffusion encoding. When an echo planar imaging (EPI) readout is used in combination with the symmetric bipolar waveform, the unavoidable deadtime between the excitation and refocusing pulses means that this waveform is not optimal in terms of echo time.
Various other diffusion encoding waveforms that use an asymmetric design to optimize the echo time have recently been proposed [15-20]. The ODGD [19] and CODE [16] formulations use constrained optimization algorithms to find arbitrarily shaped echo time optimized diffusion encoding waveforms.

When using asymmetric diffusion encoding waveforms (an example of one such waveform is given in Fig. 1c), spatially dependant concomitant gradient fields, which are characterized by Maxwell's equations, can induce dephasing and therefore signal loss over the field of view in DWI sequences $[25,26]$. These concomitant gradients must therefore be accounted for either in the pulse sequence design or with postprocessing. One issue with both the symmetric and asymmetric motion-compensated $m_{1}$-nulled waveforms is that the vessel suppression capability is decreased and $T_{2}$ shine through is increased $[15,20]$. Therefore, vessels in liver DWI acquired with motion-compensated waveforms can appear bright, which can confound lesion detection and cause errors in ADC quantification. To address the bright vessel signal problem, adding $m_{1}>0$ back to the motioncompensated waveforms (sym-vc or asym-vc) has been proposed to find a balance between motion sensitivity and vessel signal suppression $[15,20,27,28]$. An example of one such asymmetric partial velocity-compensated waveform is given in Fig. 1d.
Fig. 1 Diffusion encoding waveform designs for pgse, sym-vc, asym-vc and asym-pvc. sym-vc is symmetric and therefore does not have an optimal echo time. asym-vc is optimized for echo time by making the lobes asymmetrical. asym-pvc takes the m1-nulled design of asym-vc and adds small pgse gradients (at $b$-values other than the maximum $b$-value) to increase the $m_{1}$ value. At the highest $b$-value, the increased $\mathrm{m}_{1}$ is achieved by adjusting the relative lengths of each lobe

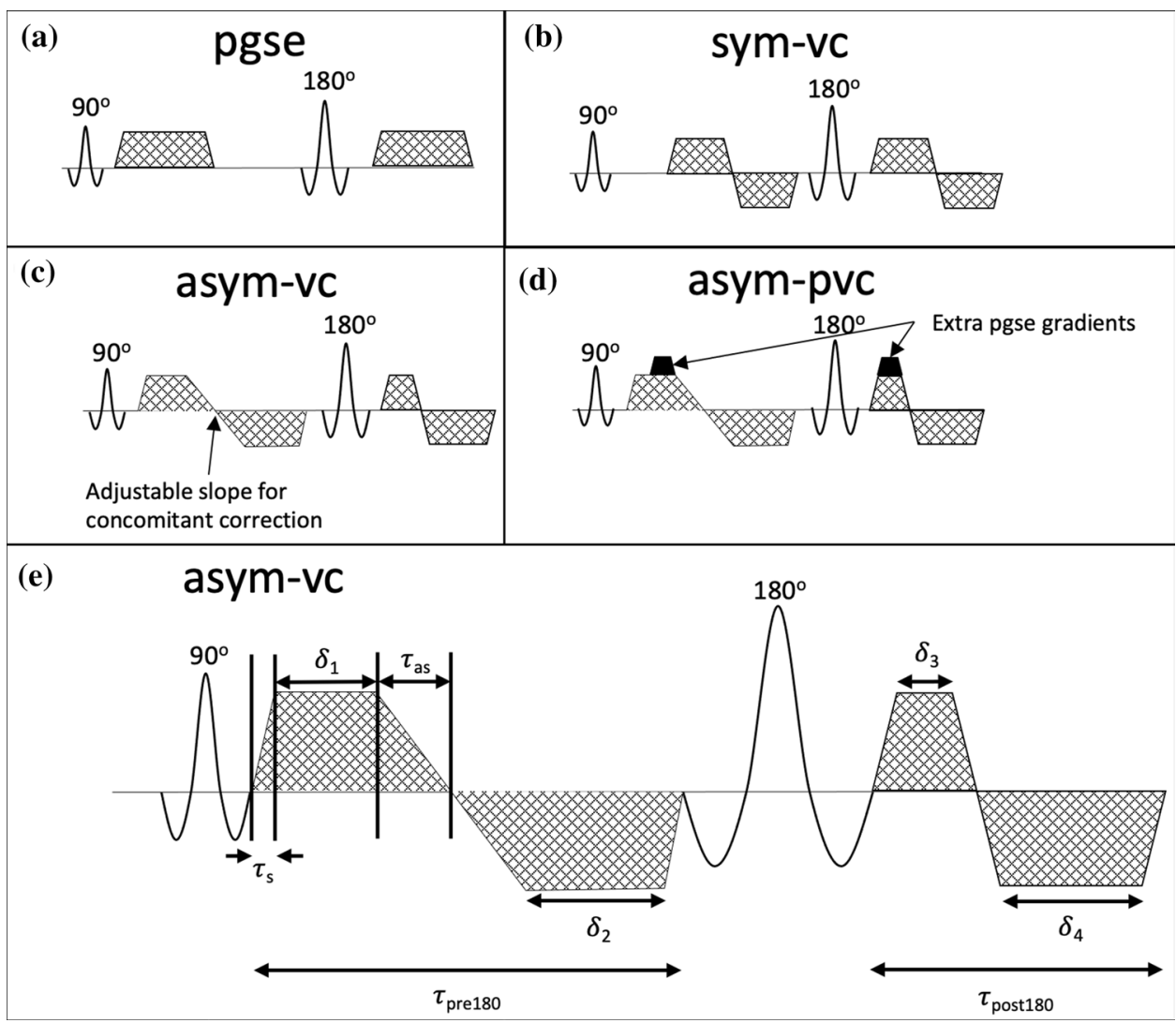


Another source of acquisition-related errors in ADC estimation is gradient nonlinearity, which is a static effect specific to the gradient coil design. With imaging gradients, gradient nonlinearity deviates the relationship between the spatial position and acquired data frequencies, leading to spatial image warping [29, 30]. With diffusion gradients, gradient nonlinearity causes the $b$-value to be different than intended and can cause ADC quantification errors larger than $10 \%$ in clinically relevant field of views [31]. Specifically, since the calculation of the $b$-value in DWI relies on a spatially uniform diffusion-weighted gradient, any spatially non-uniform gradient leads to errors in the calculation of the $b$-value and, therefore, to ADC bias [31].

Previous works have proposed methods to correct for gradient nonlinearity effects in diffusion MRI [31-34]. Bammer et al. formulated a general mathematical framework characterizing errors from gradient nonlinearity [32, 33]. The gradient field was approximated with a spherical harmonic expansion, which was then used to derive a gradient nonlinearity tensor, enabling the correction of both the magnitude and direction of the gradients. Although comprehensive, the above approach requires the acquisition of at least six diffusion directions. This is commonplace in diffusion tensor imaging (DTI) experiments, but in many body diffusion applications DWI protocols based on three orthogonal directions are typically acquired. Malyarenko et al. have proposed a simplified scheme to correct ADC bias due to gradient nonlinearity in DWI protocols employing three orthogonal directions [33]. The scope of the correction proposed by Malyarenko et al. was limited to DWI applications and was, therefore, not suitable for DTI applications [33]. The correction involved the rotation of the gradient nonlinearity tensor into the diffusion gradient frame, where the spatial bias of the $b$-matrix was approximated by the Euclidean norm. Although it has been theoretically predicted to work for arbitrary waveform designs, none of the previous works to correct ADC bias due to gradient nonlinearity in body DWI have been experimentally applied to any diffusion encoding gradient waveforms other than the conventional monopolar Stejskal-Tanner pgse diffusion encoding gradient waveform. Therefore, it is yet to be experimentally verified that the gradient nonlinearity correction method based on the work by Malyarenko et al. will be equally as effective for motioncompensated diffusion encoding waveforms as for the conventional monopolar diffusion encoding waveform.

The aim of the present work is to assess the effectiveness of the gradient nonlinearity correction for different motioncompensated diffusion encoding waveforms and specifically to characterize the effectiveness of the gradient nonlinearity correction in removing ADC bias due to gradient nonlinearity for different motion-compensated diffusion encoding waveforms in the presence of motion effects in the context of ADC mapping in the liver.

\section{Methods}

\section{Motion-compensated diffusion encoding waveforms}

The following diffusion encoding waveforms were presently investigated (Fig. 1).

\section{Pulsed gradient spin echo waveform (pgse)}

The standard monopolar Stejskal-Tanner pgse diffusion encoding waveform (Fig. 1a) was first used as it remains the most frequently used diffusion encoding waveform in body DWI.

\section{Symmetric velocity-compensated waveform (sym-vc)}

In the symmetric bipolar waveform (sym-vc), shown in Fig. $1 \mathrm{~b}$, the gradient first moment $\left(m_{1}=\gamma \int t G(t) d t\right)$ is zero.

\section{Asymmetric velocity-compensated waveform (asym-vc)}

When an echo planar imaging (EPI) readout is used in combination with the symmetric bipolar waveform, the unavoidable deadtime between the excitation and refocusing pulses means that this waveform is not optimal in terms of echo time. An asymmetric velocity-compensated diffusion encoding waveform with optimized echo time was then used. The adoption of asymmetric diffusion encoding waveforms to minimize the echo time requires the additional consideration of concomitant gradient effects, which do not cancel out as in the case of symmetric diffusion encoding waveforms $[25,35,36]$. Different strategies have been previously performed to define velocity-compensated diffusion encoding with optimal TE and correction of concomitant field effects, including strategies that approximate the concomitant phase as a linear phase variation [16] and strategies that null the concomitant gradient-induced phase accrual [19]. Specifically, by optimizing the $b$-value formulation for arbitrary gradient waveforms with appropriate constraints on system hardware, sequence timings, concomitant fields, and gradient moments, the optimized diffusion-weighting gradient waveform design (ODGD) defined a TE-optimized concomitant field corrected motion-compensated waveform [19]. However, since the optimization for the ODGD waveform must typically be done offline (not at the scanner), a file that contains the optimized waveform has to be loaded onto the scanner, reducing in general the flexibility for protocol optimization.

The presently employed asymmetric velocity-compensated (asym-vc) waveform builds upon the ODGD formulation [19]. Without concomitant field correction, the optimal ODGD 
waveform is always a collection of trapezoids. Additionally, for a wide range of relevant sequence parameters in the employed $b$-value range, the number of trapezoid lobes and polarities of an optimized waveform do not change. Therefore, the optimization of the waveform can be reduced to the optimization of the trapezoid lengths, which can be integrated into the pulse sequence environment and carried out efficiently online at the scanner. When concomitant field correction is added, the ODGD formulation yields optimal waveforms that are not trapezoidal. Alternatively, by making the slope of the trapezoidal waveform a variable and adding the concomitant field correction as a constraint, first-order concomitant field correction is applied by adjusting the slopes of the trapezoids until the concomitant field term is nulled. This second concomitant correction approach is similar to Zhang et al. [15] and Szczepankiewicz et al. [23, 24] and is suitable for the waveform optimization at the scanner.

The design of the asym-vc waveform is as follows. The asym-vc waveform is assumed to have four lobes, two before and two after the refocusing pulse. Figure 1e shows a labelled diagram of the asym-vc waveform, where $\delta_{1}, \delta_{2}, \delta_{3}$ and $\delta_{4}$ are the durations of the plateaus of each lobe, $\tau_{\mathrm{s}}$ is the duration of the non-adjustable slope, $\tau_{\mathrm{as}}$ is the duration of the adjustable slope and $\tau_{\text {pre180 }}$ and $\tau_{\text {post } 180}$ are the durations available before and after the refocusing pulse, respectively. The non-adjustable slopes are constant and are equal to the maximum slew rate. The other constraints for the timing of each lobe are the $m_{0}$, $m_{1}$ and concomitant constraints. The variables to solve for, in terms of the amount of time before and after the refocusing pulse, are the duration of each plateau of each lobe and the slope in between the first and second lobes. The constraints are therefore

$\delta_{1}+\delta_{2}+2 \tau_{\mathrm{s}}+2 \tau_{\mathrm{as}}=\tau_{\mathrm{pre} 180}$,

$\delta_{3}+\delta_{4}+4 \tau_{\mathrm{s}}=\tau_{\text {post } 180}$

$m_{0}=\gamma \int_{\tau} G(t) d t=0$

$m_{1}=\gamma \int_{\tau} t G(t) d t=0$

$\gamma \int_{A 1} G(t)^{2} d t-\gamma \int_{A 2} G(t)^{2} d t=0$

where the integrals of $m_{0}$ and $m_{1}$ are over the entire duration of the gradient waveforms, given by $\tau$, and $A 1$ and $A 2$ are the time periods before and after the refocusing pulse, respectively. Equation 5 is the constraint imposed to minimize concomitant fields. With these five constraints, the five variables $\delta_{1}, \delta_{2}, \delta_{3}, \delta_{4}$ and $\tau_{\text {as }}$ can be found.
To obtain the asym-vc waveform, the $b$-value equation is expressed in terms of the gradient lobe timings, which is then input into the scanner software environment and the echo time is optimized for a given $b$-value. $\tau_{\text {pre180 }}$ and $\tau_{\text {post } 180}$ are found from each so-called "target echo time" in the optimization loop. As the asym-vc waveform is restricted to trapezoidal waveforms and not arbitrarily shaped waveforms, the optimization procedure is not burdened by long computational times unsuitable for the direct scanner interface. Therefore, asym-vc waveform can be designed on-the-fly on the scanner, instead of having to input a text file containing the offline optimized waveform whenever scan parameters are changed. The resulting waveform has an asymmetric bipolar design as shown in Fig. 1c.

A further description about the differences between the implementation of the asym-vc, and representatives of existing optimized velocity-compensated waveforms such as the ODGD and the CODE [16] waveforms are included in the Supplementary Material.

\section{Asymmetric partially velocity-compensated waveform (asym-pvc)}

Compared to the pulse field gradient encoding (pgse) waveform, the sym-vc and asym-vc waveforms, while decreasing sensitivity to motion, also decrease the vessel suppression capability and increase $T_{2}$ shine through $[15,20]$. Therefore, vessels in liver DWI acquired with sym-vc and asymvc can appear bright, which can confound lesion detection and cause errors in ADC quantification. The fact that the vessels can appear bright is caused in part by nulling the gradient first moment, $m_{1}$. By adding a small $m_{1}>0$ back to the motion-compensated waveforms (sym-vc or asymvc) significant reduction of vessel signals can be achieved $[15,20,27,28]$. The key idea of these methods is to find an appropriate $m_{1}>0$ value that balances sensitivity to motion and vessel signal suppression. In the present work, small pgse gradients corresponding to the desired value of $\mathrm{m}_{1}$ were added to two of the gradient lobes of the asym-vc waveform for all $b$-values except for the maximum $b$-value, similar to Zhang et al. [15]. At the maximum $b$-value, in the present implementation, the lengths of each of the gradient lobes and the amplitude of the asym-vc gradients were adjusted until the desired value of $m_{1}$ was obtained without changing the $b$-value. Although not fully TE-optimized, the above asymmetric partial motion-compensated waveform (asym-pvc) can be again designed on-the-fly at the scanner, facilitating protocol optimization. The asym-pvc waveform is shown in Fig. 1d. A detailed analysis of the employed asymmetric partially velocity-compensated waveform design 
is provided in the supplementary material, with a diagram shown in Fig. S1.

\section{Gradient nonlinearity correction}

The gradient nonlinearity correction (gnl) framework as proposed by Malyarenko et al. was adopted [33]. The method is briefly described here: in the case of linear gradients, the gradient strength is uniform across the whole magnet bore and does not depend on position. In the case of nonlinear gradients, the gradient strength is dependent on position within the magnet bore and spurious gradients orthogonal to the applied gradient will be produced. First-order spatial variations of the gradient can be described by a nonlinearity tensor $\boldsymbol{L}(\boldsymbol{r})$, where

$\boldsymbol{g}(\boldsymbol{r})=\boldsymbol{L}(\boldsymbol{r}) \boldsymbol{g}_{0}$, wherel $_{i j}(\boldsymbol{r})=\frac{1}{\left\|g_{i 0}\right\|} \frac{\partial B_{z}^{g_{i}}}{\partial r_{j}},\left(\boldsymbol{L} \neq \boldsymbol{L}^{T}\right)$,

where $\boldsymbol{g}_{0}$ is the gradient at isocenter, $\boldsymbol{r}$ is the spatial position from isocenter and $\boldsymbol{g}(\boldsymbol{r})$ is the spatially varying gradient. $\partial B_{z}^{g_{i}}$ is the $z$ component of the magnetic field as a result of applying the gradient in the $i$ direction and $g_{i 0}$ is the $i$ component of the gradient $g_{0}$. The spatially dependent magnetic field produced by each gradient coil can be described by a spherical harmonic expansion. Using the spherical harmonic coefficients provided by the manufacturer, the spatially varying magnetic field produced by each coil and, therefore, the gradient nonlinearity tensor $\boldsymbol{L}$ can be derived. The spatially varying b matrix is given by $\boldsymbol{b}^{\prime}(\boldsymbol{r})=\boldsymbol{L}(\boldsymbol{r}) \boldsymbol{b}_{0} \boldsymbol{L}^{T}(\boldsymbol{r})$, where $\boldsymbol{b}_{0}$ is the nominal $b$ matrix and includes $b$ components due to the diffusion gradients, imaging gradients and imaging cross terms. The correction map is then obtained from (with further details given in [33])
$C^{k}(\boldsymbol{r})=\frac{\left\|\boldsymbol{b}^{\boldsymbol{\prime}^{k}}(\boldsymbol{r})\right\|_{F}}{\left\|\boldsymbol{b}^{k}\right\|_{F}} \cong \frac{\sqrt{\operatorname{Tr}\left\{\left[\boldsymbol{b}^{\boldsymbol{\prime}^{k}}(\boldsymbol{r})\right]^{2}\right\}}}{b_{0}^{k}} \cong\left[\boldsymbol{u}_{k}^{T} \boldsymbol{L}(\boldsymbol{r}) \boldsymbol{u}_{k}\right]\left[\boldsymbol{u}_{k}^{T} \boldsymbol{L}^{T}(\boldsymbol{r}) \boldsymbol{u}_{k}\right]$,

for each diffusion direction $k$, where $\operatorname{Tr}\{$.$\} is the trace$ operator, $F$ is the Frobenius norm and $\boldsymbol{u}_{k}$ is a unit vector that defines the $k$ th DW direction in the gradient coil coordinates. The corrected $b$-value map is given by

$b_{c}^{k}(\boldsymbol{r})=b_{n}^{k} C^{k}(\boldsymbol{r})$,

where $b_{c}^{k}(\boldsymbol{r})$ is the corrected spatially dependent $b$-value map for each diffusion direction $\mathrm{k}, b_{n}^{k}$ is the nominal $b$-value and $C^{k}(\boldsymbol{r})$ is the correction map. Example correction maps are given in Fig. 2.

The code used to calculate the correction maps was provided by the vendor and was integrated into the reconstruction platform on the scanner. The individual $b$-values and directions as well as the design parameters of the gradient coil and slice orientations were input to the reconstruction process, after which the correction maps were generated as a reconstruction output of the diffusion scans. The ADC maps were computed in MATLAB from the correction maps and the diffusion-weighted data exported from the scanner.

\section{MRI measurements}

All MRI measurements were performed on a $3 \mathrm{~T}$ Ingenia Elition X scanner (Philips Healthcare, Best, Netherlands), which has a maximum gradient amplitude of $45 \mathrm{mT} / \mathrm{m}$ and a maximum slew rate of $200 \mathrm{~T} / \mathrm{m} / \mathrm{s}$.
$\mathrm{RO}(\mathrm{x})$

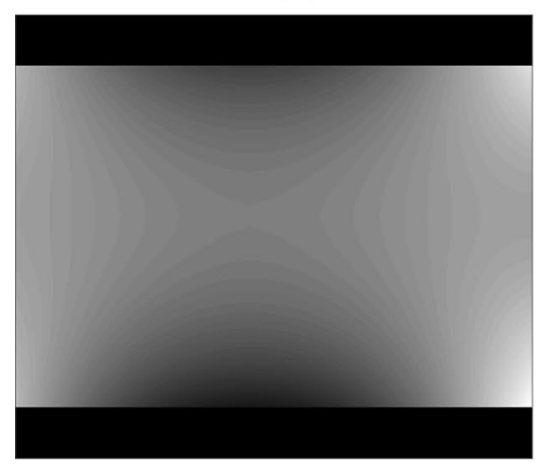

PE $(z)$

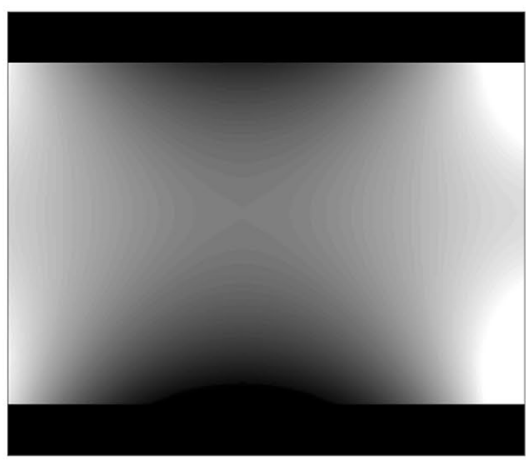

SS (y)

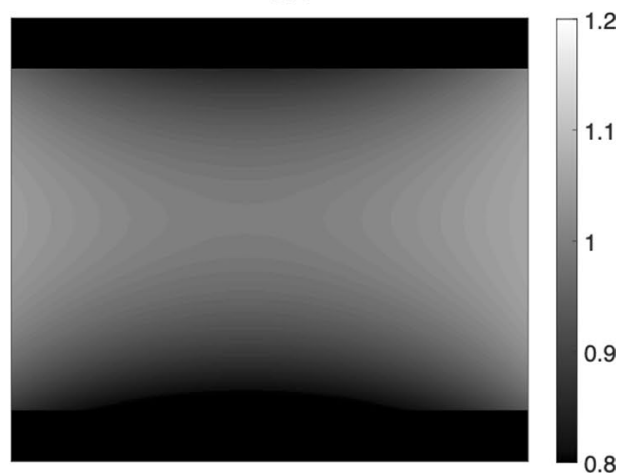

Fig. $2 b$-value correction maps for diffusion directions RO, PE and $\mathrm{SS}$ from one coronal slice of one of the in vivo measurements. The scale shows the ratio between the corrected and uncorrected $b$-value.
The same correction maps were used for all waveforms. The scanner axes are given in brackets 


\section{Phantom measurements}

Phantom validation measurements were performed to assess the performance of the gradient nonlinearity correction with the pgse, sym-vc, asym-vc and asym-pvc waveforms. A cylindrical phantom of length $200 \mathrm{~mm}$ and diameter $115 \mathrm{~mm}$ containing $31.5 \mathrm{mmol} / \mathrm{L}$ of $\mathrm{NiCl}_{2}-6 \mathrm{H}_{2} \mathrm{O}$ was placed inside an extremity coil at isocenter and axial slices were acquired with a ss-EPI sequence in the S/I direction to investigate the gradient nonlinearity in the $\mathrm{S} / \mathrm{I}$ direction. A separate scan in which sagittal slices in the $\mathrm{L} / \mathrm{R}$ direction were acquired to investigate the gradient nonlinearity in the $\mathrm{L} / \mathrm{R}$ direction was performed. More information is given in the supplementary material. Gradient nonlinearity was not measured in the $\mathrm{A} / \mathrm{P}$ direction due to the unsuitability of the dimensions of the phantom for measuring in this direction.

For all waveforms in the axial slice experiment, seventeen slices were acquired with a FoV of $125 \times 125 \mathrm{~mm}^{2}$, a voxel size of $2 \times 2 \times 6 \mathrm{~mm}^{3}$ and a $2.4 \mathrm{~mm}$ slice gap. The parallel imaging acceleration factor $R=2$, effective bandwidth over phase encode direction $=57 \mathrm{~Hz} /$ pixel. $B$-values of $[0,600] \mathrm{s} / \mathrm{mm}^{2}$ were used for the ADC calculation, with eight averages per $b$-value and three orthogonal diffusion encoding directions along the readout (RO), phase encoding (PE) and slice selection (SS) directions. The echo times were 60/90/90/96 ms for pgse, asym-vc, asym-pvc and sym$\mathrm{vc}$, respectively. $\mathrm{TR}=8000 \mathrm{~ms}$ in all cases and no partial Fourier encoding was used for any of the waveforms. An $\mathrm{m}_{1}$ value of $0.1 \mathrm{~s} / \mathrm{mm}$ was used for the asym-pvc waveform.

ADC maps were calculated for each individual diffusion direction, as well as for all directions combined. An ROI was drawn in the center of each slice and the mean ADC over the ROI was calculated.

A separate scan was performed to test the performance of the concomitant gradient correction. The same phantom was placed inside the extremity coil and seventeen axial slices were acquired with a FoV of $128 \times 128 \mathrm{~mm}^{2}$, a voxel size of $2 \times 2 \times 6 \mathrm{~mm}^{3}$ and a $2.5 \mathrm{~mm}$ slice gap. This scan was performed without parallel imaging and also with a parallel imaging factor of $R=2$. When a parallel imaging factor of $R=2$ is used, the number of acquired $k$-space lines is reduced in comparison to when no parallel imaging is used. Therefore, the time interval available for diffusion encoding after the refocusing pulse is closer to the time interval before the refocusing pulse, meaning that the diffusion encoding waveforms before and after the refocusing pulse are more symmetrical. Since concomitant gradient effects are affected by the asymmetry of the waveforms, using a parallel imaging factor of $R=2$ was expected to reduce the effect of the concomitant gradients. For both scans, $\mathrm{TR}=5000 \mathrm{~ms}, b$-values of $[0,600] \mathrm{s} / \mathrm{mm}^{2}$ were used for the ADC calculation, with five averages per $b$-value and three orthogonal diffusion encoding directions along the readout (RO), phase encoding (PE) and slice selection (SS) directions. For the scan without parallel imaging, effective bandwidth over phase encode direction $=27.2 \mathrm{~Hz} /$ pixel, the echo times were $98 / 103 \mathrm{~ms}$ for asym-vc with and without concomitant gradient correction, respectively. For the scan with a parallel imaging factor of $R=2$, effective bandwidth over phase encode direction $=54.5 \mathrm{~Hz} /$ pixel, the echo times were $89 / 90 \mathrm{~ms}$ for asym-vc with and without concomitant gradient correction, respectively.

Using identical scan parameters apart from a TR of $6000 \mathrm{~ms}$, an asym-pvc scan with an $\mathrm{m}_{1}$ value of $0.1 \mathrm{~s} / \mathrm{mm}$ was performed with a parallel imaging factor of $R=2$ and $b$-values of $[0,600,600] \mathrm{s} / \mathrm{mm}^{2}$, with each of the $b=600 \mathrm{~s} /$ $\mathrm{mm}^{2}$ acquisitions using a different waveform design for inducing $m_{1}$. Waveform 1 is the design of the lower $b$-values, with a separate pgse gradient added on top of the existing asym-vc gradient to increase the $m_{1}$. Waveform 2 is the design of the higher $b$-value, meaning that $m_{1}$ is added by changing the relative lengths of the gradient lobes of the asym-vc gradients. The above experiment was done to validate that both waveform designs for inducing $m_{1}$ give the same ADC estimation.

\section{In vivo measurements}

In vivo measurements were carried out in 4 healthy volunteers (mean age, $34 \pm 6$ years) using the built in 12-channel posterior and 16-channel anterior coil. The study was approved by the local ethics commission and all volunteers have consented for their participation in the study.

Two slices of the liver were acquired in the coronal plane with a FoV of $240 \times 312 \mathrm{~mm}^{2}$, a voxel size of $3 \times 3 \times 6 \mathrm{~mm}^{3}$, parallel imaging acceleration factor $R=3$, effective bandwidth over phase encode direction $=53.3 \mathrm{~Hz} /$ pixel, number of packages $=1, b$-values of $[200,600] \mathrm{s} / \mathrm{mm}^{2}$ and corresponding averages of $[3,4]$. The $b$-value of $200 \mathrm{~s} / \mathrm{mm}^{2}$ was chosen to minimize any perfusion signal contributions to the ADC estimation. To reduce echo time for better SNR, diffusion encoding directions $[-0.5-1-1]$, [1 $0.5-1]$, [ $\left.\begin{array}{lll}1 & -1 & 0.5\end{array}\right]$ were used. These diffusion encoding directions (which shall be referred to from now on as the overplus diffusion directions) were used for all four subjects, giving echo times of 53/80/80/90 ms for pgse, asym-vc, asym-pvc and sym-vc respectively. The TR was determined by the breathing and cardiac cycle of each volunteer, and was in the range of approximately 3000-6000 ms.

For three out of the four subjects, scans with diffusion encoding along the RO, PE and SS directions (magnet diffusion directions) were additionally performed, giving echo times of 60/88/88/94 ms for pgse, asym-vc, asym-pvc and sym-vc, respectively. This was not done for the fourth subject due to time constraints and the fact that this subject 
had lower liver signal, meaning that the increased echo time would have reduced the image quality.

Scans with both cardiac and respiratory triggering (double triggering), as well as scans with only respiratory triggering were performed for both sets of diffusion directions (magnet and overplus). The imaging parameters were identical between the double-triggered and respiratory-triggered scans.

ADC maps were generated, and subtraction maps were computed from subtracting the ADC maps without any gradient nonlinearity correction from the ADC maps with gradient nonlinearity correction. For the ROI analysis, ROIs were drawn on one slice of the pgse ADC map from the respiratory-triggered scans. These ROIs were then propagated to the subtraction maps for each diffusion encoding waveform. One of the ROIs was drawn in the left liver lobe in a region with an overestimated ADC due to motion corruption, taking care to avoid large vessels. This ROI was chosen to investigate interactive effects of both motion and gradient nonlinearity on the ADC estimation. The other ROI was drawn close to the right inferior side of the liver.

\section{Correction maps}

Figure 2 shows correction maps of the gradient nonlinearity correction for the RO, PE and SS diffusion directions. The correction maps calculated from the gradient field spherical harmonics for each diffusion direction were equal between the different diffusion encoding waveforms.

\section{Results}

\section{Phantom results}

Figure 3 shows the results from the phantom experiment in which slices were acquired in the S/I direction (gradient nonlinearity in the S/I direction). Without the gradient
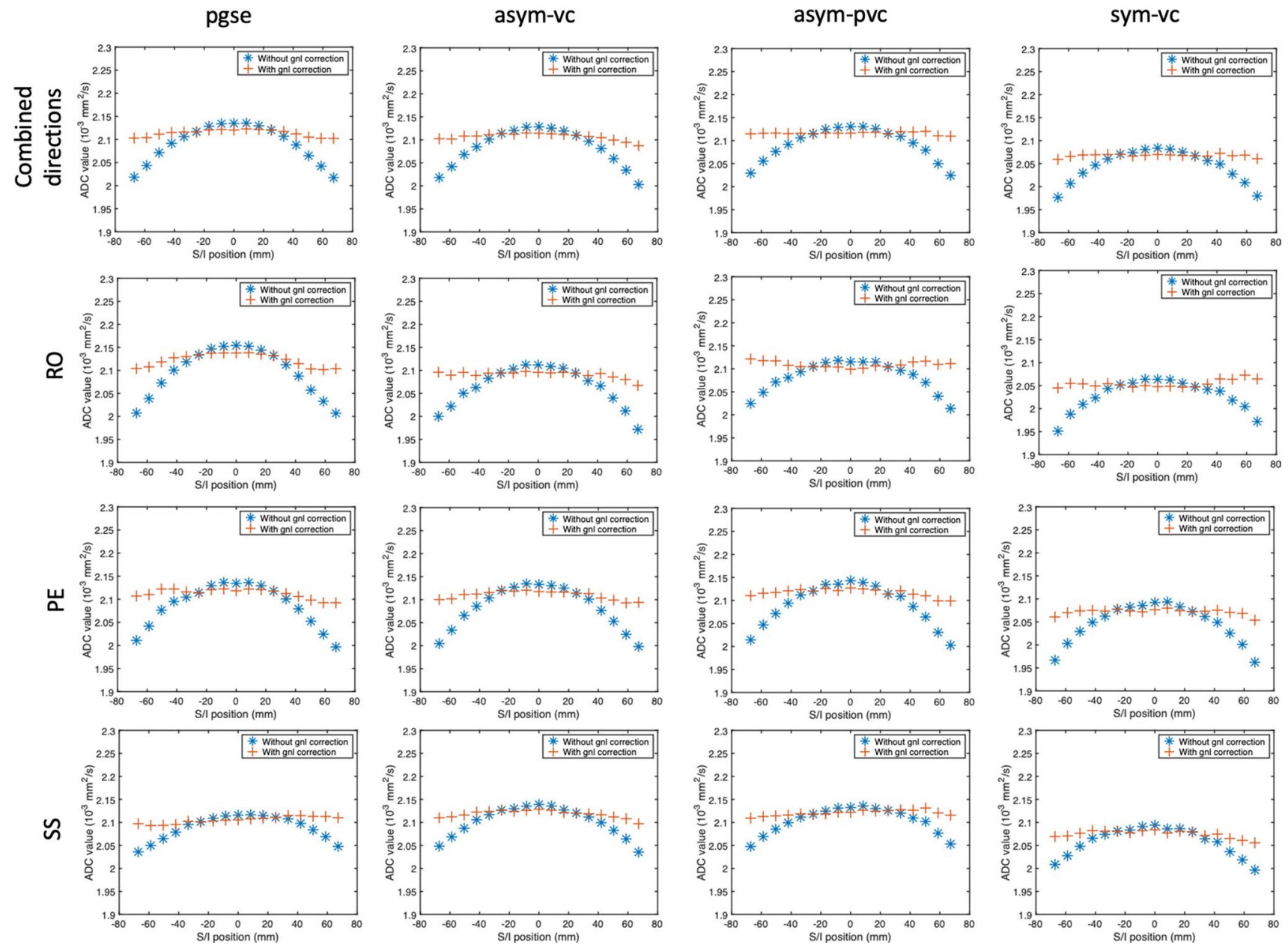

Fig. 3 Gradient nonlinearity along the S/I direction. The curve of the non-corrected ADC for an ROI drawn in the center of the phantom shows the characteristic gradient-nonlinearity-induced shape. After correction, this bias is considerably reduced for all waveforms 
nonlinearity correction, along the S/I direction, the average ADCs exhibit the characteristic gradient nonlinearity curve, in which a local maximum or minimum is at the isocenter, and the mean ADC increases or decreases symmetrically away from the isocenter. After the gradient nonlinearity correction, the pgse waveform yields minimal variation of $\mathrm{ADC}$ for each diffusion direction. Before gradient nonlinearity correction, the standard deviation of the ADC (calculated from all directions combined) across all slices is $4.36 \times 10^{-5}$ $\mathrm{mm}^{2} / \mathrm{s}$. After gradient nonlinearity correction, this is $1.02 \times 10^{-5} \mathrm{~mm}^{2} / \mathrm{s}$. The range of the ADC values for pgse over a distance of $\pm 67.2 \mathrm{~mm}$ from isocenter is $1.29 \times 10^{-4}$ $\mathrm{mm}^{2} / \mathrm{s}$ before gnl correction, and $0.32 \times 10^{-4} \mathrm{~mm}^{2} / \mathrm{s}$ after gnl correction. Similar to the pgse waveform, the asym-vc, asym-pvc and sym-vc waveforms also show the characteristic ADC variation when no gradient nonlinearity correction is performed. With the gradient nonlinearity correction, the curves were flattened for all waveforms. The asym-vc waveform has a standard deviation of ADC (calculated from all directions combined) across all slices of $4.08 \times 10^{-5} \mathrm{~mm}^{2} / \mathrm{s}$ before gnl correction, and $0.75 \times 10^{-5} \mathrm{~mm}^{2} / \mathrm{s}$ after gnl correction. The range of the ADC values for asym-vc over a distance of $\pm 67.2 \mathrm{~mm}$ from isocenter is $1.22 \times 10^{-4} \mathrm{~mm}^{2} / \mathrm{s}$ before gnl correction, and $0.24 \times 10^{-4} \mathrm{~mm}^{2} / \mathrm{s}$ after gnl correction. The sym-vc waveform has a standard deviation of ADC (calculated from all directions combined) across all slices of $3.16 \times 10^{-5} \mathrm{~mm}^{2} / \mathrm{s}$ before gnl correction, and $0.60 \times 10^{-5} \mathrm{~mm}^{2} / \mathrm{s}$ after gnl correction. The range of the ADC values for sym-vc over a distance of $\pm 67.2 \mathrm{~mm}$ from isocenter is $1.04 \times 10^{-4} \mathrm{~mm}^{2} / \mathrm{s}$ before gnl correction, and $0.22 \times 10^{-4} \mathrm{~mm}^{2} / \mathrm{s}$ after gnl correction. The asym-pvc waveform shows a slight overcompensation in the RO direction, as the curve looks inverted compared to the case without gradient nonlinearity correction; however, the variation in ADC is still reduced considerably. This waveform has a standard deviation of ADC (calculated from all directions combined) across all slices of $3.54 \times 10^{-5} \mathrm{~mm}^{2} / \mathrm{s}$ before gnl correction, and $0.26 \times 10^{-5} \mathrm{~mm}^{2} / \mathrm{s}$ after gnl correction. The range of the ADC values for asym-pvc over a distance of $\pm 67.2 \mathrm{~mm}$ from isocenter is $1.07 \times 10^{-4} \mathrm{~mm}^{2} / \mathrm{s}$ before $\mathrm{gnl}$ correction, and $0.11 \times 10^{-4} \mathrm{~mm}^{2} / \mathrm{s}$ after gnl correction. For all waveforms, with or without gradient nonlinearity correction, any observable dependence of the ADC variation on the diffusion encoding direction is small. The results from the phantom experiment in which slices were acquired in the $\mathrm{R} / \mathrm{L}$ direction (gradient nonlinearity in the $\mathrm{R} / \mathrm{L}$ direction) are shown in the supplementary material Fig. S2.

\section{Concomitant gradient phantom experiment}

A simulation of the effect of concomitant gradients on the ADC value in a phantom is given in the Supplementary Material. The difference in ADC for a range of distances from the isocenter is given in the Supplementary Material Fig. S4.

Figure 4 shows the phantom results for the asym-vc waveform with and without concomitant gradient correction for two different parallel imaging factors. When no
Fig. 4 asym-vc waveform with and without concomitant gradient correction. When no parallel imaging factor is used $(R=1)$, there is a considerable overestimation of the ADC on one side of the CG-uncorrected curve after gnl correction.

When a parallel imaging factor of $2(R=2)$ is used, the number of acquired $k$-space lines is reduced in comparison to when no parallel imaging is used. Therefore, the time interval available for diffusion encoding after the refocusing pulse is closer to the time interval before the refocusing pulse, meaning that the diffusion encoding waveforms before and after the refocusing pulse are more symmetrical, and the effect of concomitant gradients is therefore not as large. The CG-corrected waveform has a fairly constant ADC across the FoV after gnl correction
CG-uncorrected
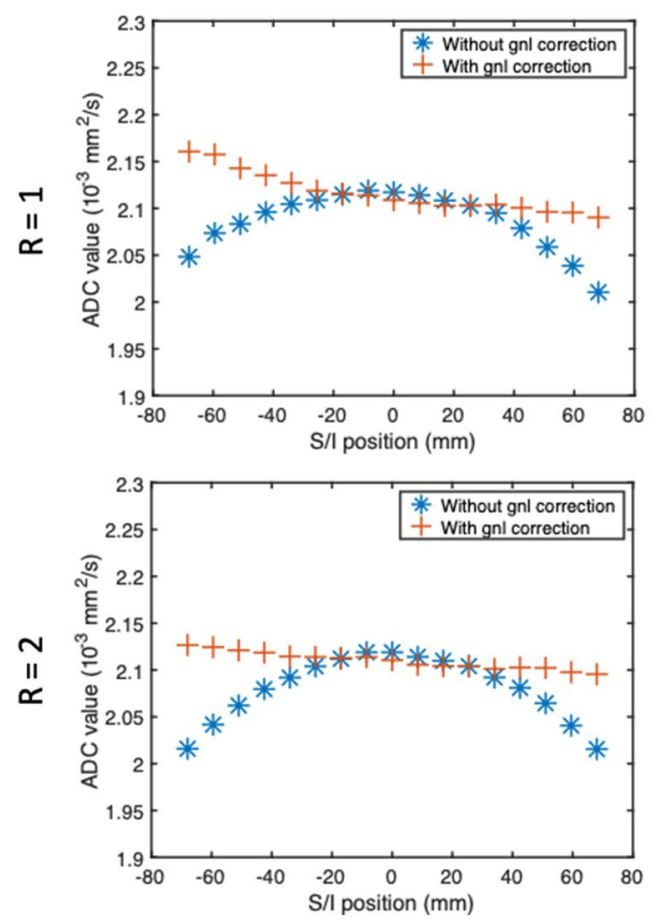

CG-corrected
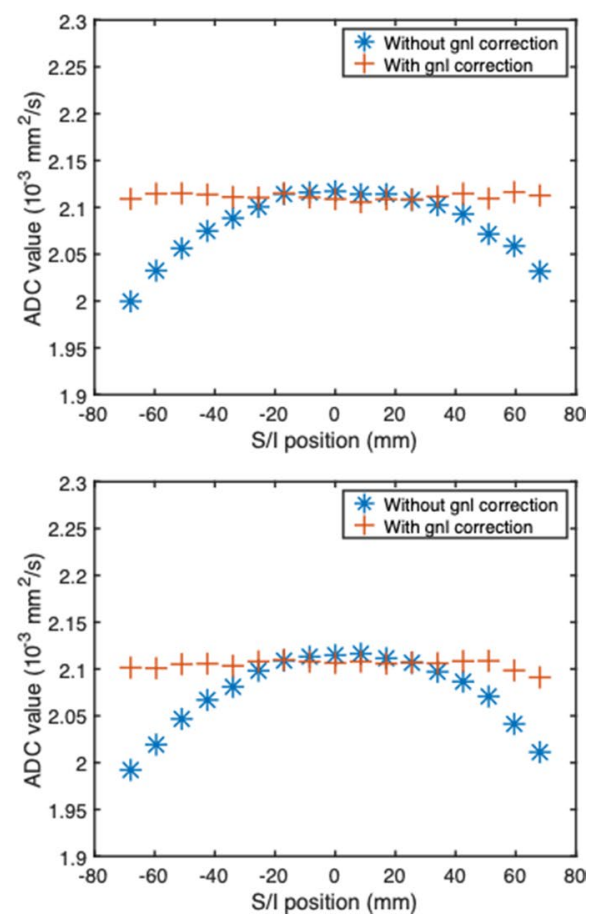
parallel imaging is used, the difference in ADC between a slice approximately $60 \mathrm{~mm}$ away from isocentre and a slice at isocentre was approximately $4.9 \times 10^{-5} \mathrm{~s} / \mathrm{mm}^{2}$. When a parallel imaging factor of 2 was used, this ADC difference was $1.4 \times 10^{-5} \mathrm{~s} / \mathrm{mm}^{2}$. When no parallel imaging was used, the standard deviation of the ADC of the asym-vc with concomitant correction was $3.55 \times 10^{-5} \mathrm{~s} / \mathrm{mm}^{2}$ before gnl correction, and $0.30 \times 10^{-5} \mathrm{~s} / \mathrm{mm}^{2}$ after gnl correction. The standard deviation of the ADC of the asym-vc without concomitant correction was $3.14 \times 10^{-5} \mathrm{~s} / \mathrm{mm}^{2}$ before gnl correction, and $2.14 \times 10^{-5} \mathrm{~s} / \mathrm{mm}^{2}$ after gnl correction, indicating that the CG correction is performing well. When a parallel imaging factor of 2 was used, meaning that the waveform was less asymmetric and therefore would not have as severe concomitant gradient effects, the standard deviation of the ADC of the asym-vc without concomitant correction was $3.48 \times 10^{-5} \mathrm{~s} / \mathrm{mm}^{2}$ before gnl correction, and $0.93 \times 10^{-5} \mathrm{~s} /$ $\mathrm{mm}^{2}$ after gnl correction. The standard deviation of the ADC of the asym-vc with concomitant correction was $3.99 \times 10^{-5} \mathrm{~s} / \mathrm{mm}^{2}$ before gnl correction, and $0.47 \times 10^{-5} \mathrm{~s} /$ $\mathrm{mm}^{2}$ after gnl correction, again remaining stable.

\section{asym-pvc waveform comparison}

Figure 5 shows the phantom results for the comparison between the two different waveforms used to make up asympvc. The maximum difference in ADC between the two waveforms at the same slice was $0.66 \times 10^{-5} \mathrm{~s} / \mathrm{mm}^{2}$ before gnl correction, and $0.65 \times 10^{-5} \mathrm{~s} / \mathrm{mm}^{2}$ after gnl correction.

\section{In vivo results}

Figure 6 shows the uncorrected ADC maps, corrected ADC maps, subtraction maps and correction maps for two different scans performed with the pgse waveform, one with double cardiac and respiratory triggering, and one with only respiratory triggering. It is clear that in the left liver lobe region when only respiratory triggering is used, the ADC is overestimated. In the subtraction maps, the left liver lobe region also has high values (with a mean ADC difference of $1.4 \times 10^{-5} \mathrm{~mm}^{2} / \mathrm{s}$ for the respiratory-triggered scans, and $1.0 \times 10^{-5} \mathrm{~mm}^{2} / \mathrm{s}$ for the double-triggered scans), indicating that the gradient nonlinearity correction has increased the motion-induced overestimated ADC further. ADC maps and subtraction maps from two volunteers are shown in the supplementary material Fig. S5.

Figure 7 shows the uncorrected ADC maps, corrected ADC maps, subtraction maps and correction maps for all waveforms in which only respiratory triggering was used. The diffusion encoding directions were the overplus directions for the first volunteer, and RO, PE and SS for the second volunteer. The ADC in the left liver lobe when the pgse waveform was used is again overestimated. The subtraction map shows that the gradient nonlinearity correction has increased the motion-induced overestimated ADC even further. The motion-compensation capability of the asym-pvc, asym-vc and sym-vc waveforms reduces the motion-induced overestimation of ADC at the cost of lower SNR, compared to when the pgse waveform was used. Subtraction maps show lower values in the left liver lobe for asym-pvc, asymvc and sym-vc waveforms compared to the pgse waveform. Since bright vessels will also have a high ADC, this is also further increased by the gradient nonlinearity correction in certain regions, depending on the correction map. This effect can also be seen in the subtraction maps. The effect of gnl correction on overestimated ADC is explained further in the supplementary material Fig. S3. ADC maps and subtraction maps from two volunteers are shown in the supplementary material Fig. S6.

Figure 8 shows the ROI analysis performed on the subtraction maps for all subjects for all waveforms. The plots show the mean value of the subtraction map over the ROI, and the error bars show the standard deviation over the ROI. In all subjects, the asym-pvc, asym-vc and sym-vc waveforms had a lower mean subtraction map value in the left liver lobe region compared to the pgse waveform. In the
Fig. 5 asym-pvc waveform $\left(m_{1}=0.1 \mathrm{~s} / \mathrm{mm}\right)$ with ADC calculated from both of the waveform designs. Waveform 1 is the design of the lower $b$-values, with a separate pgse gradient added on top of the existing asym- $v c$ gradient to increase the $m_{1}$. Waveform 2 is the design of the higher $b$-value, meaning that $m_{1}$ is added by changing the relative lengths of the gradient lobes of the asym-vc gradients. Both waveforms give almost identical ADC values gnl-uncorrected

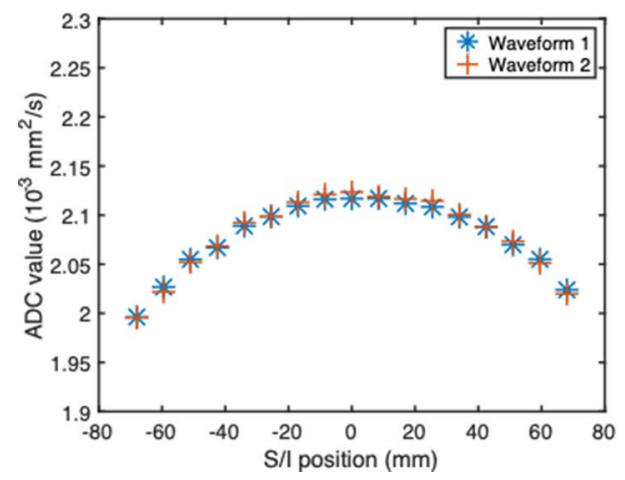

gnl-corrected

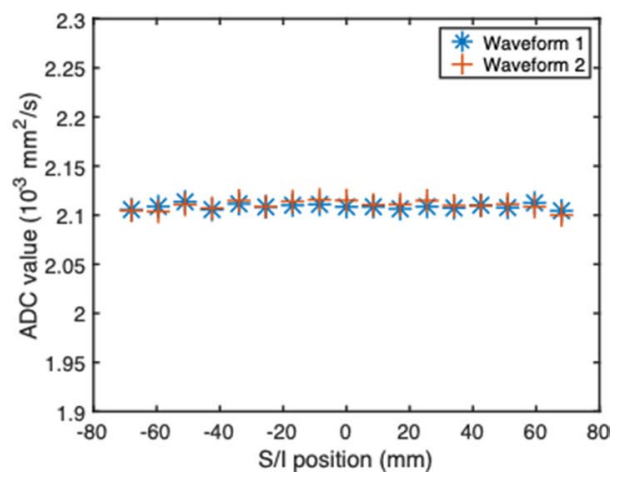


Fig. 6 Uncorrected ADC maps, corrected ADC maps, subtraction maps (calculated by subtracting the uncorrected ADC map from the corrected ADC map) and correction maps for the pgse waveform with double triggering and only respiratory triggering for one volunteer. The respiratory-triggered case shows a clear overestimation of the ADC in the left liver lobe. The subtraction map shows a positive value in this region, highlighting an interaction between motion effects and gnl correction. The gnl correction will increase an already overestimated $\mathrm{ADC}$ in this region, as shown in the corrected ADC maps
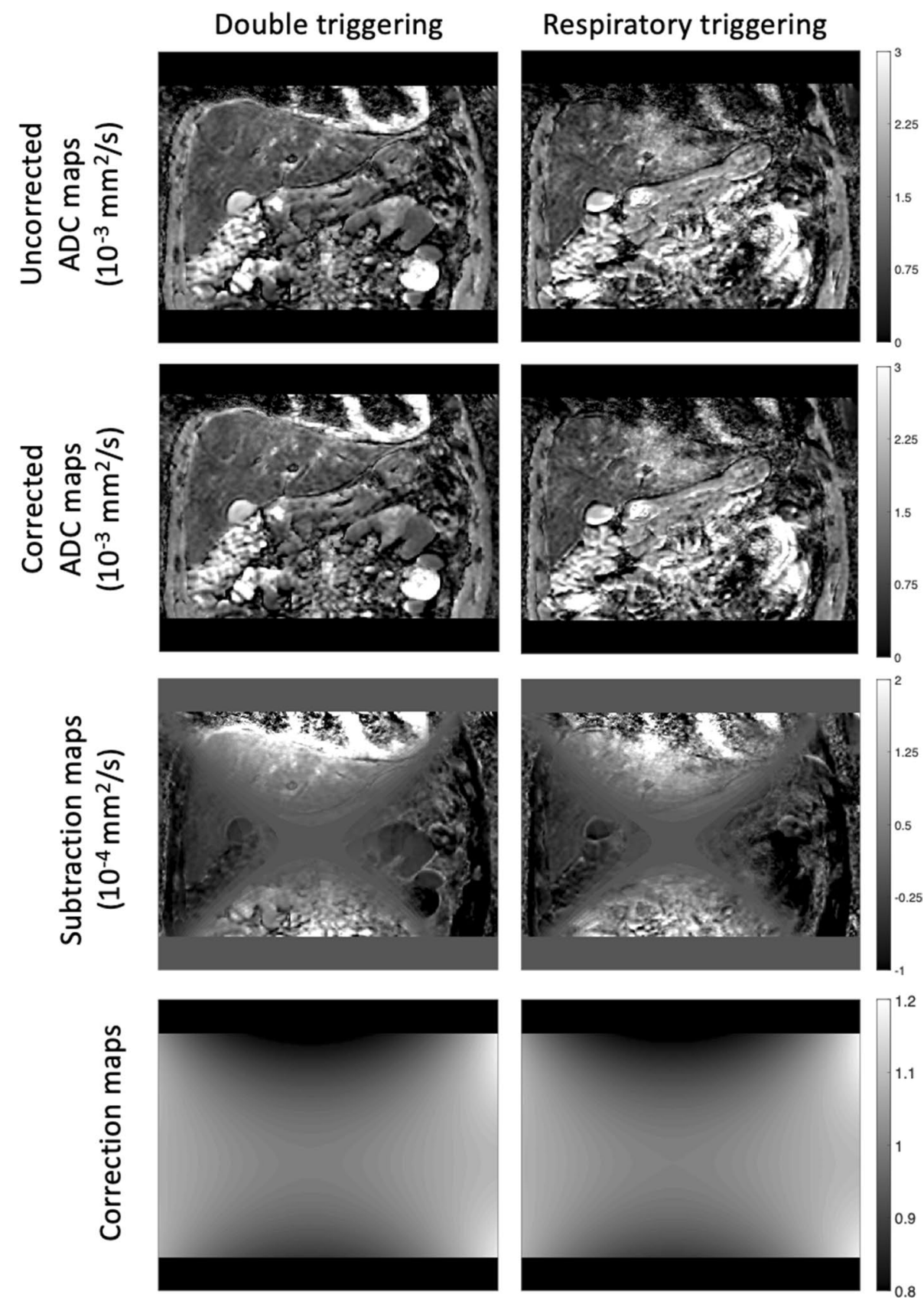

right liver lobe region, the differences between all waveforms are typically smaller.

\section{Discussion}

The present work applies the gradient nonlinearity correction using the spherical harmonic expansion of the gradient coil field [33] to different motion-compensated diffusion encoding waveforms. Despite known disadvantages such as the prolonged echo time and reduced SNR, occurrence of bright vessel signal, concomitant effects if asymmetrical waveforms are used, motion-compensated diffusion encoding waveforms remain an alternative approach to more accurate ADC quantification. The gradient nonlinearity correction has, to the best of our knowledge, only been applied to the standard monopolar pgse diffusion encoding waveform and the present work, therefore, showed the feasibility of using the gradient nonlinearity correction with motion-compensated diffusion encoding waveforms.

Although not the main focus of the present study, the present work also proposes methods for online computation of near-TE-optimal motion-compensated and partial 

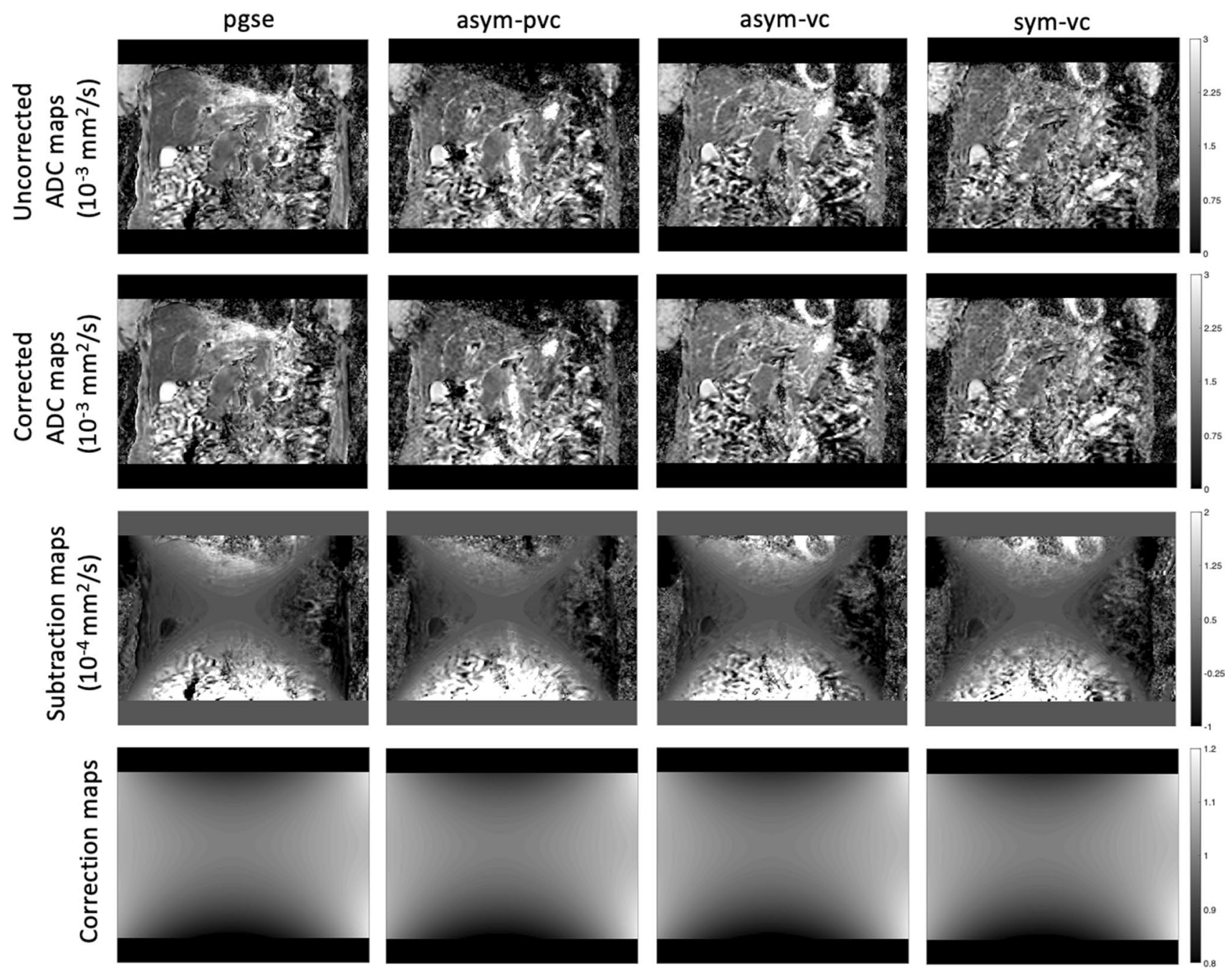

Fig. 7 Uncorrected ADC maps, corrected ADC maps, subtraction maps and correction maps for all waveforms. The data were acquired with diffusion encoding along the overplus directions. The correction maps were the same for all waveforms. The uncorrected ADC maps for the motion-compensated waveforms do not show as large of an ADC overestimation in the left liver lobe when compared with pgse.

motion-compensated waveforms with concomitant field correction, allowing flexibility in protocol optimization [37, 38]. The presently employed methods utilize the knowledge from the offline TE-optimized gradient waveform design as proposed by Peña-Nogales et al. [19]. However, the implementation of the gradient nonlinearity correction was found to be applicable to different motion-compensated waveform designs, and should also be applicable to any diffusion encoding waveform designed offline or on-the-fly as in $[37,38]$. The asym-pvc achieved partial velocity compensation by adjusting the lengths of the lobes for the maximum $b$-value, and by adding small pgse gradients to the asym-vc waveform for the lower $b$-values. The difference in design
The subtraction maps therefore do not show as large of a difference between the gnl corrected and non-corrected ADC maps, apart from in regions where there are large vessels. The corrected ADC maps for the motion-compensated waveforms also do not show as large of an $\mathrm{ADC}$ overestimation in the left liver lobe when compared with pgse

between the $b$-values was done to mimic the design of Zhang et al. [15] and for ease of implementation.

Figure 2 shows the correction maps for the RO, PE and SS directions. Since the employed correction procedure holds for a general gradient waveform in which the polarity is reversed at TE/2 [33, 39] (to implicitly account for the effect of the spin echo RF pulse [40]), the correction maps were the same for all waveforms.

The phantom results show that the gradient nonlinearity correction can considerably reduce ADC bias for all waveforms. All waveforms showed some residual fluctuation after correction, which can be attributed to higher order gradient nonlinearity effects, or other factors such as eddy currents or residual concomitant gradient effects and is a 

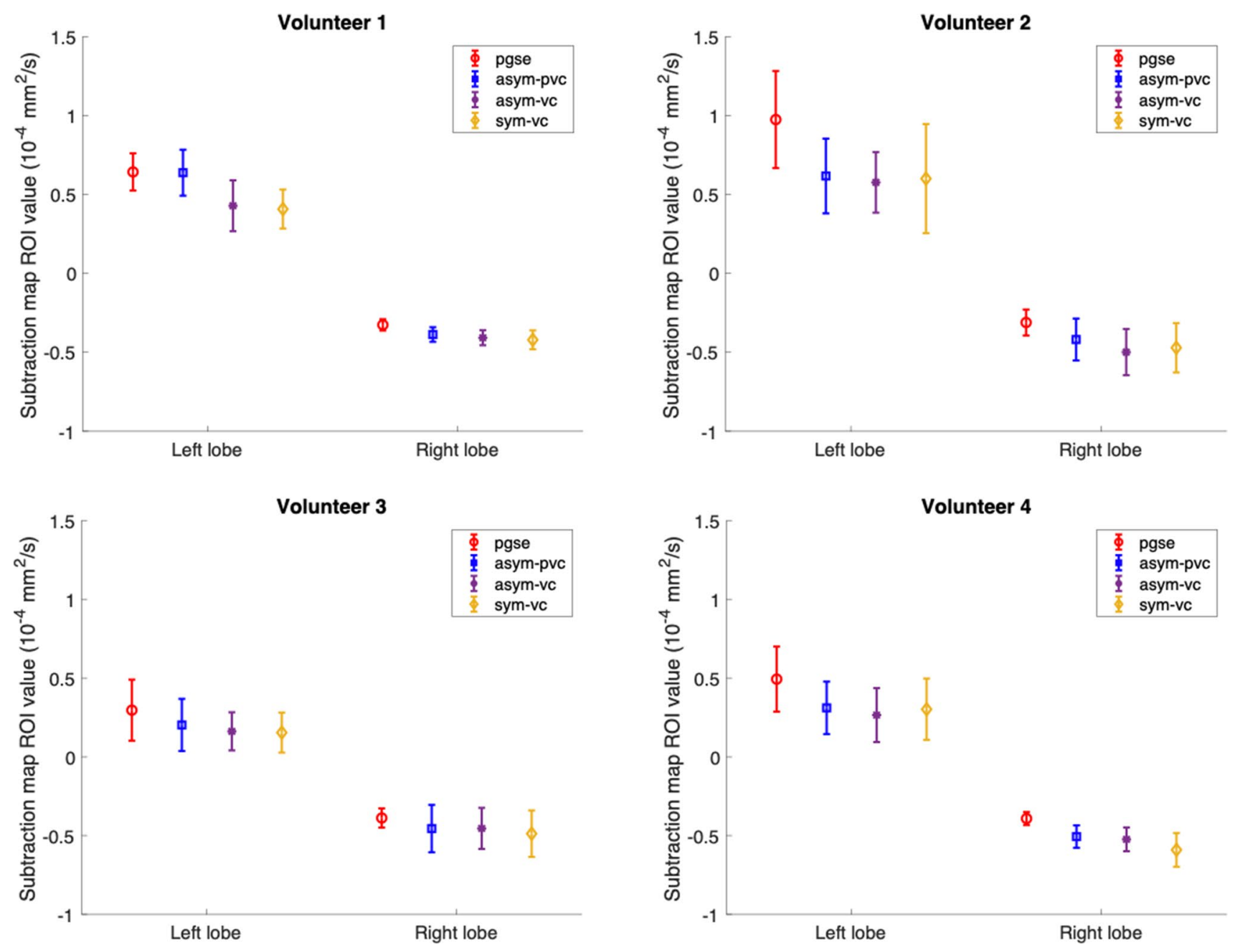

Fig. 8 Subtraction map ROI analysis for all subjects in which an ROI was drawn in the left liver lobe in a region that had been corrupted by motion and another ROI was drawn in the right inferior region of the liver. In the left lobe region, the motion-compensated waveforms have

a lower mean value in the subtraction map ROI, possibly as a result of the decreased sensitivity to motion. The differences between the waveforms in the right liver lobe region, which is not heavily affected by motion, are typically smaller

subject for further investigation. The asym-pvc waveform showed a slight overcompensation in the RO direction, as the curve looks inverted compared to the case without gradient nonlinearity correction. This could be a result of residual concomitant gradient effects. When the current nonlinear gradient correction method is employed, similar levels of residual fluctuation of ADC values were observed across all waveforms, suggesting that the employed method can correct for gradient nonlinearity just as well in the case of the more complicated motion-compensated waveforms.

The gnl correction can be affected by non-gnl related ADC errors. Evidence of such interactions between gnl correction and non-gnl related ADC errors was shown in Figs. 6 and 8 in the left liver lobe and regions with bright vessels.

The phantom experiment for the asym-vc concomitant gradient correction in Fig. 4 showed that even when no parallel imaging factor was used, therefore making the

waveform more asymmetric and increasing the impact of the concomitant fields, the CG-corrected waveform remained stable. The CG-uncorrected waveform showed larger deviations in ADC away from isocentre when no parallel imaging factor was used. When a parallel imaging factor of 2 was used, the variation in ADC was lower; however, it was still larger than the CG-corrected waveform, indicating that the concomitant correction performed well.

The phantom experiment for the two different waveform designs for asym-pvc in Fig. 5 showed that the ADC calculated from both waveform designs was almost identical, justifying the usage of using different designs for the lower and higher $b$-values in the asym-pvc waveform.

The subtraction map ROI analysis in Fig. 8 showed that for all subjects, the pgse waveform had a higher subtraction map value in the left liver lobe in a region which has overestimated ADC due to motion. The higher subtraction 
map value highlights the interaction between the gradient nonlinearity correction and ADC overestimation. In the right liver lobe region, the differences between the pgse waveform and the motion-compensated waveforms were smaller, as this is a region that is not as heavily affected by motion. The asym-pvc waveform also typically had a higher subtraction map value in the left liver lobe than the asym-vc waveform, which could be due to the fact that the increase in $\mathrm{m}_{1}$ also increases sensitivity to motion. In the right liver lobe region, the motion-compensated waveforms also had a larger standard deviation over the ROI in the subtraction map than pgse, which could be explained by the lower SNR and bright vessel signal. Therefore, the presented results suggest that to get accurate ADC quantification, one has to take gradient nonlinearity, motion sensitivity, vessel signal suppression and the impact of these on each other into account.

The present work has some limitations. First, the phantom measurements were performed without any temperature control. In Malyarenko et al. [33], an ice water phantom was used to keep the temperature constant throughout the experiment. Second, only a distance of $\pm 67.2 \mathrm{~mm}$ from isocentre was measured with the presently used phantom. Despite these limitations, the pgse results without the gradient nonlinearity correction showed the characteristic curve of ADC bias, which was flattened by the gradient nonlinearity correction. Third, only four healthy volunteers were scanned in the present work. Further work would be required to fully characterize the interaction between gradient nonlinearityinduced and motion-induced bias in liver ADC quantification of subjects with different motion patterns and liver ADC variations.

\section{Conclusion}

The gradient nonlinearity correction based on the spherical harmonic expansion of the gradient coil field was used in conjunction with different motion-compensated waveforms, thereby showing the feasibility of the usage of the gradient nonlinearity correction with gradient waveforms other than the standard monopolar pgse diffusion encoding waveform. The phantom results showed a reduction in ADC bias in all waveforms after the gradient nonlinearity correction was applied. The in vivo results showed an interaction between the gradient nonlinearity correction, ADC overestimation in the left liver lobe due to motion, and bright vessel signal from the motion-compensated waveforms. This suggests that to get accurate $\mathrm{ADC}$ quantification, one has to take gradient nonlinearity, motion sensitivity, vessel signal suppression and the impact of these on each other into account.
Supplementary Information The online version contains supplementary material available at https://doi.org/10.1007/s10334-021-00981-6.

Acknowledgements The present work was supported by Philips Healthcare. The authors would like to thank Dr. Thomas Chenevert and Dr. Dariya Malyarenko for helpful discussions.

Author contributions All authors contributed to the study conception and design. Material preparation, data collection and analysis were performed by SM. The first draft of the manuscript was written by SM and all authors commented on previous versions of the manuscript. All authors read and approved the final manuscript.

Funding Open Access funding enabled and organized by Projekt DEAL.

\section{Declarations}

Conflict of interest Johannes M. Peeters and Kilian Weiss are employees of Philips Healthcare. Dimitrios C. Karampinos receives grant support from Philips Healthcare.

Ethics approval The study was approved by the local ethics commission and all volunteers have consented for their participation in the study.

Open Access This article is licensed under a Creative Commons Attribution 4.0 International License, which permits use, sharing, adaptation, distribution and reproduction in any medium or format, as long as you give appropriate credit to the original author(s) and the source, provide a link to the Creative Commons licence, and indicate if changes were made. The images or other third party material in this article are included in the article's Creative Commons licence, unless indicated otherwise in a credit line to the material. If material is not included in the article's Creative Commons licence and your intended use is not permitted by statutory regulation or exceeds the permitted use, you will need to obtain permission directly from the copyright holder. To view a copy of this licence, visit http://creativecommons.org/licenses/by/4.0/.

\section{References}

1. Namimoto T, Yamashita Y, Sumi S, Tang Y, Takahashi M (1997) Focal liver masses: characterization with diffusion-weighted echoplanar MR imaging. Radiology 204:739-744

2. Ichikawa T, Haradome H, Hachiya J, Nitatori T, Araki T (1998) Diffusion-weighted MR imaging with a single-shot echoplanar sequence: detection and characterization of focal hepatic lesions. Am J Roentgenol 170:397-402

3. Kwee TC, Takahara T, Koh DM, Nievelstein RA, Luijten PR (2008) Comparison and reproducibility of ADC measurements in breathhold, respiratory triggered, and free-breathing diffusion-weighted MR imaging of the liver. J Magn Reson Imaging 28(5):1141-1148

4. Kandpal H, Sharma R, Madhusudhan KS, Kapoor KS (2009) Respiratory-triggered versus breath-hold diffusion-weighted MRI of liver lesions: comparison of image quality and apparent diffusion coefficient values. Am J Roentgenol 192(4):915-922

5. Taouli B, Sandberg A, Stemmer A, Parikh T, Wong S, Xu J, Lee VS (2009) Diffusion-weighted imaging of the liver: comparison of navigator triggered and breathhold acquisitions. J Magn Reson Imaging 30(3):561-568 
6. Eatesam M, Noworolski SM, Tien PC, Nystrom M, Dodge JL, Merriman RB, Qayyum A (2012) Liver diffusivity in healthy volunteers and patients with chronic liver disease: comparison of breathhold and free-breathing techniques. J Magn Reson Imaging 35(1):103-109

7. Kwee TC, Takahara T, Niwa T, Ivancevic MK, Herigault G, Van Cauteren M, Luijten PR (2009) Influence of cardiac motion on diffusion-weighted magnetic resonance imaging of the liver. MAGMA 22(5):319-325

8. Murphy P, Wolfson T, Gamst A, Sirlin C, Bydder M (2013) Error model for reduction of cardiac and respiratory motion effects in quantitative liver DW-MRI. Magn Reson Med 70(5):1460-1469

9. Nasu K, Kuroki Y, Sekiguchi R, Nawano S (2006) The effect of simultaneous use of respiratory triggering in diffusion-weighted imaging of the liver. Magn Reson Med Sci 5(3):129-136

10. Bruegel M, Holzapfel K, Gaa J, Woertler K, Waldt S, Kiefer B, Stemmer A, Ganter C, Rummeny EJ (2008) Characterization of focal liver lesions by ADC measurements using a respiratory triggered diffusion-weighted single-shot echo-planar MR imaging technique. Eur Radiol 18(3):477-485

11. Gourtsoyianni S, Papanikolaou N, Yarmenitis S, Maris T, Karantanas A, Gourtsoyiannis N (2008) Respiratory gated diffusionweighted imaging of the liver: value of apparent diffusion coefficient measurements in the differentiation between most commonly encountered benign and malignant focal liver lesions. Eur Radiol 18(3):486-492

12. Mürtz P, Flacke S, Träber F, van den Brink JS, Gieseke J, Schild HH (2002) Abdomen: diffusion-weighted MR imaging with pulsetriggered single-shot sequences. Radiology 224(1):258-264

13. Metens T, Absil J, Denolin V, Bali MA, Matos C (2016) Liver apparent diffusion coefficient repeatability with individually predetermined optimal cardiac timing and artifact elimination by signal filtering. J Magn Reson Imaging 43(5):1100-1110

14. Hahn S, Absil J, Debeir O, Metens T (2019) Assessment of cardiac-driven liver movements with filtered harmonic phase image representation, optical flow quantification, and motion amplification. Magn Reson Med 81(4):2788-2798

15. Zhang Y, Peña-Nogales Ó, Holmes JH, Hernando D (2019) Motion-robust and blood suppressed M1-optimized diffusion MR imaging of the liver. Magn Reson Med 82:302-311

16. Aliotta E, Wu HH, Ennis DB (2017) Convex optimized diffusion encoding (CODE) gradient waveforms for minimum echo time and bulk motion compensated diffusion weighted MRI. Magn Reson Med 77:717-729

17. Majeed W, Kalra P, Kolipaka A (2018) Motion Compensated, Optimized Diffusion Encoding (MODE) Gradient Waveforms. In: Proceedings of the 26th Annual Meeting of ISMRM. Paris, France: Abstract 1614

18. Ozaki M, Inoue Y, Miyati T, Hata H, Mizukami S, Komi S, Matsunaga K, Woodhams R (2013) Motion artifact reduction of diffusion-weighted MRI of the liver: use of velocity-compensated diffusion gradients combined with tetrahedral gradients. J Magn Reson Imaging 37(1):172-178

19. Peña-Nogales Ó, Zhang Y, Wang X, de Luis-Garcia R, AjaFernández S, Holmes JH, Hernando D (2019) Optimized Diffusion-Weighting Gradient Waveform Design (ODGD) formulation for motion compensation and concomitant gradient nulling. Magn Reson Med 81:989-1003

20. Van AT, Cervantes B, Ogino T, Peeters JM, Hock A, Rummeny EJ, Braren R, Karampinos DC (2018) Partial velocity-compensated diffusion encoding for combined motion compensation and residual vessel signal suppression in liver DWI. In: Proceedings of the 26th Annual Meeting of ISMRM. Paris, France: Abstract 0079

21. Sjölund J, Szczepankiewicz F, Nilsson M, Topgaard D, Westin CF, Knutsson H (2015) Constrained optimization of gradient waveforms for generalized diffusion encoding. J Magn Reson 261:157-168

22. Szczepankiewicz F, Sjölund J (2021) Cross-term-compensated gradient waveform design for tensor-values diffusion MRI. J Magn Reson 328:106991

23. Szczepankiewicz F, Sjölund J, Dall'Armellina E, Plein S, Schneider JE, Teh I, Westin CF (2021) Motion-compensated gradient waveforms for tensor-values diffusion encoding by constrained numerical optimization. Magn Reson Med 85:2117-2126

24. Szczepankiewicz F, Westin CF, Nilsson M (2019) Maxwell-compensated design of asymmetric gradient waveforms for tensorvalued diffusion encoding. Magn Reson Med 82:1424-1437

25. Baron CA, Lebel RM, Wilman AH, Beaulieu C (2012) The effect of concomitant gradient fields on diffusion tensor imaging. Magn Reson Med 68(4):1190-1201

26. Szczepankiewicz F, Eichner C, Anwander A, Westin CF, Paquette M (2020) The impact of gradient non-linearity on Maxwell compensation when using asymmetric gradient waveforms for tensorvalued diffusion encoding. In: Proceedings of the 28th Annual Meeting of ISMRM. Abstract 3391

27. McTavish S, Van AT, Peeters J, Ogino T, Hock A, Rummeny E, Braren R, Karampinos DC (2019) Partial velocity-compensated optimized diffusion encoding for combined motion compensation and residual vessel signal suppression in liver ADC mapping. In: Proceedings of the 27th Annual Meeting of ISMRM. Montréal, Canada: Abstract 1709

28. Rauh SS, Riexinger AJ, Ohlmeyer S, Hammon M, Saake M, Stemmer A, Uder M, Hensel B, Laun FB (2020) A mixed waveform protocol for reduction of the cardiac motion artifact in black-blood diffusion-weighted imaging of the liver. Magn Reson Imaging 67:59-68

29. Janke A, Zhao H, Cowin GJ, Galloway GJ, Doddrell DM (2004) Use of spherical harmonic deconvolution methods to compensate for nonlinear gradient effects on MRI images. Magn Reson Med 52(1):115-122

30. Jovicich J, Czanner S, Greve D, Haley E, van der Kouwe A, Gollub R, Kennedy D, Schmitt F, Brown G, Macfall J, Fischl B, Dale A (2006) Reliability in multi-site structural MRI studies: effects of gradient non-linearity correction on phantom and human data. Neuroimage 30(2):436-443

31. Malkyarenko DI, Chenevert TL (2014) Practical estimate of gradient nonlinearity for implementation of apparent diffusion coefficient bias correction. J Magn Reson Imaging 40(6):1487-1495

32. Bammer R, Markl M, Barnett A, Acar B, Alley MT, Pelc NJ, Glover GH, Moseley ME (2003) Analysis and generalized correction of the effect of spatial gradient field distortions in diffusionweighted imaging. Magn Reson Med 50(3):560-569

33. Malyarenko DI, Ross BD, Chenevert TL (2014) Analysis and correction of gradient nonlinearity bias in apparent diffusion coeffcient measurements. Magn Reson Med 71:1312-1323

34. Tan ET, Marinelli L, Slavens ZW, King KF, Hardy CJ (2013) Improved correction for gradient nonlinearity effects in diffusionweighted imaging. J Magn Reson Imaging 38(2):448-453

35. Bernstein MA, Zhou XJ, Polzin JA, King KF, Ganin A, Pelc NJ, Glover GH (1998) Concomitant gradient terms in phase contrast MR: analysis and correction. Magn Reson Med 39(2):300-308

36. Meier C, Zwanger M, Feiweier T, Porter D (2008) Concomitant field terms for asymmetric gradient coils: consequences for diffusion, flow, and echo-planar imaging. Magn Reson Med 60(1):128-134

37. Loecher M, Middione MJ, Ennis DB (2020) A gradient optimization toolbox for general purpose time-optimal MRI gradient waveform design. Magn Reson Med 84(6):3234-3245

38. Middione MJ, Loecher M, Moulin K, Ennis DB (2020) Optimization methods for magnetic resonance imaging gradient waveform design. NMR Biomed 33(12):4308 
39. Gullmar D, Haueisen J, Reichenbach JR (2005) Analysis of b-value calculations in diffusion weighted and diffusion tensor imaging. Concepts Magn Res A 25:53-66

40. Stejskal EO, Tanner JE (1965) Spin diffusion measurements: spin echoes in the presence of a time-dependent field gradient. J Chem Phys 42:288-292
Publisher's Note Springer Nature remains neutral with regard to jurisdictional claims in published maps and institutional affiliations. 